\title{
Quantitative prediction of transient and steady-state elongational viscosity of nearly monodisperse polystyrene melts
}

\author{
Wagner, Manfred H.; Kheirandish, Saeid; Hassager, Ole
}

Published in:

Journal of Rheology

Link to article, DOI:

$10.1122 / 1.2048741$

Publication date:

2005

Document Version

Publisher's PDF, also known as Version of record

Link back to DTU Orbit

Citation (APA):

Wagner, M. H., Kheirandish, S., \& Hassager, O. (2005). Quantitative prediction of transient and steady-state elongational viscosity of nearly monodisperse polystyrene melts. Journal of Rheology, 49(6), 1317-1327. https://doi.org/10.1122/1.2048741

\section{General rights}

Copyright and moral rights for the publications made accessible in the public portal are retained by the authors and/or other copyright owners and it is a condition of accessing publications that users recognise and abide by the legal requirements associated with these rights.

- Users may download and print one copy of any publication from the public portal for the purpose of private study or research.

- You may not further distribute the material or use it for any profit-making activity or commercial gain

- You may freely distribute the URL identifying the publication in the public portal 


\title{
Quantitative prediction of transient and steady-state elongational viscosity of nearly monodisperse polystyrene melts
}

\author{
Manfred H. Wagner ${ }^{\text {a) }}$ and Saeid Kheirandish \\ Polymertechnik / Polymerphysik, Technische Universität Berlin \\ Fasanenstrasse 90, D-10623 Berlin, Germany \\ Ole Hassager \\ The Danish Polymer Center, Department of Chemical Engineering, \\ Technical University of Denmark, DK-2800 Kgs. Lyngby, Denmark
}

(Received 18 May 2005; final revision received 1 August 2005)

\begin{abstract}
Synopsis
Elongational behavior of four narrow molar mass distribution polystyrene melts of masses 50000 , 100 000, 200 000, and 390 000, g/mol, respectively was investigated up to Hencky strains of 5. All melts show strain hardening behavior. For the two highest molar mass polystyrenes, strain hardening starts at elongation rates larger than the inverse reptation time, and the steady-state elongational viscosities decrease with increasing elongation rate according to a power law with a power-law index of approximately $-1 / 2$ instead of -1 as predicted by the original Doi-Edwards tube model. Marrucci and Ianniruberto [Macromolecules 37, 3934 (2004)] have introduced an interchain pressure term arising from lateral forces between the chain and the tube wall into the Doi-Edwards model to account for the latter effect. Based on the molecular stress function theory allowing for a strain-dependent tube diameter, we show that the transient and steady-state elongational viscosities of the nearly monodisperse polystyrene melts can be modeled quantitatively by assuming affine chain deformation balanced by the interchain pressure term of Marrucci and Ianniruberto. The interchain pressure is governed by a tube diameter relaxation time $\tau_{a}$, which is found to be larger than the Rouse time $\tau_{R}$ of the chain, and which is the only parameter of the model. For monodisperse polystyrene melts of sufficient low molar mass, $\tau_{a}$ is larger than the reptation time, and a maximum in the steady-state elongational viscosity is predicted. (C) 2005 The Society of Rheology. [DOI: 10.1122/1.2048741]
\end{abstract}

\section{INTRODUCTION}

Recent progress in experimental techniques to measure nonlinear properties of polymer melts and solutions has led to major breakthroughs in the rheology of polymeric systems [Schweizer (2000); McKinley and Sridhar (2002)]. Particularly, the filament stretching technique has enabled reaching elongation rates in regimes beyond the inverse Rouse time, and has revealed very interesting and unexpected aspects in steady-state

\footnotetext{
${ }^{a)}$ Author to whom correspondence should be addressed; electronic mail: manfred.wagner@tu-berlin.de
} 
elongational properties of solutions and melts of monodisperse and polydisperse polymers [see, e.g., Bhattacharjee et al. (2002) and (2003); McKinley and Sridhar (2002)].

In elongational flow, the Doi-Edwards (DE) model with the so-called independent alignment assumption predicts an upper limit of the tensile stress equal to five times the plateau modulus, $G_{N}$. This limiting stress is a consequence of the assumption of instantaneous chain retraction and, therefore, the absence of any chain stretching. According to the DE model, the macroscopic stress is a consequence of chain orientation only, resulting in a scaling of the steady-state elongational viscosity at strain rates $\dot{\epsilon}$ larger than the inverse reptation time with $\dot{\epsilon}^{-1}$. Relaxing the assumption of instantaneous chain retraction, various reptation-based models have invoked chain stretch when the deformation rate is larger than the inverse Rouse time $\tau_{R}$ of the chain [see, e.g., Pearson et al. (1989); Mead et al. (1995, 1998); Fang et al. (2000)]. However, recent elongational viscosity measurements of Bach et al. [Bach et al. (2003)] on narrow molar mass distribution polystyrene melts have revealed that chain stretch is already detectable at elongation rates larger than the inverse reptation time, and the elongational viscosity scales approximately with $\dot{\epsilon}^{-1 / 2}$. Marrucci and Ianniruberto [Marrucci and Ianniruberto (2004)] have introduced an interchain pressure term arising from lateral forces between the chain and the tube wall into the DE model to account for this effect. However, their analysis is restricted to scalar arguments and to the steady-state viscosity. Here, we present a full constitutive equation which describes time-dependent as well as steady-state rheology of nearly monodisperse polymer melts, and compare predictions to the elongational viscosity data of Bach et al. [Bach et al. (2003)] and Hassager [2004].

\section{EXPERIMENTAL DATA}

The experimental data discussed are those presented by Bach et al. [Bach et al. (2003)] and Hassager [Hassager (2004)]. Elongational viscosities of four polystyrene melts with narrow molar mass distribution were measured using a filament stretching rheometer capable of measuring at high temperatures. Most measurements were performed at $130{ }^{\circ} \mathrm{C}$, several measurements on polystyrene PS390K performed at $150{ }^{\circ} \mathrm{C}$ were shifted to $130^{\circ} \mathrm{C}$ by standard time-temperature shifting. From linear-viscoelastic mastercurves of $G^{\prime}$ and $G^{\prime \prime}$, discrete relaxation spectra with relaxation times $\lambda_{i}$ and fractional relaxation moduli $g_{i}$ were derived using the IRIS program [IRIS Development, Amherst, MA-Winter and Mours (2003)]. Polymer characterization and spectra are summarized in Table I. The Rouse time $\tau_{R}$ and the longest relaxation time $\tau_{w}$ (identified with the reptation time here) were calculated according to well-known relations [Osaki $e t$ al. (1982); Takahashi et al. (1993); Isaki et al. (2003)],

$$
\begin{gathered}
\tau_{R}=\frac{12 M \eta_{0}}{\pi^{2} \rho R T}\left(\frac{M_{c}}{M}\right)^{2.4}, \\
\tau_{w}=J_{e}^{0} \eta_{0},
\end{gathered}
$$

where $M_{c}$ of polystyrene was taken as $35000 \mathrm{~g} / \mathrm{mol}$.

\section{PREDICTIONS OF THE DOI-EDWARDS MODEL}

The intermolecular interaction of concentrated systems of linear polymer chains is modeled by the tube concept: The mesh of constraints caused by surrounding chains confines the macromolecular chain laterally to a tubelike region. Doi and Edwards assumed that the diameter $a_{0}$ of the tube is not changed even by large nonlinear deforma- 
TABLE I. Molecular characterization and discrete relaxation spectra (relaxation moduli $g_{i}$ and relaxation times $\lambda_{i}$ ) of PS samples at $130{ }^{\circ} \mathrm{C}$.

\begin{tabular}{|c|c|c|c|c|c|c|c|}
\hline \multicolumn{2}{|c|}{ PS50K } & \multicolumn{2}{|c|}{ PS100K } & \multicolumn{2}{|c|}{ PS200K } & \multicolumn{2}{|c|}{ PS390K } \\
\hline \multicolumn{2}{|c|}{$M_{w}=51700 \mathrm{~g} / \mathrm{mol}$} & \multicolumn{2}{|c|}{$M_{w}=102800 \mathrm{~g} / \mathrm{mol}$} & \multicolumn{2}{|c|}{$M_{w}=200000 \mathrm{~g} / \mathrm{mol}$} & \multicolumn{2}{|c|}{$M_{w}=390000 \mathrm{~g} / \mathrm{mol}$} \\
\hline \multirow{2}{*}{\multicolumn{2}{|c|}{$\begin{array}{c}M W D=1.10 \\
J^{0}=5.81 \times 10^{-6}\left[\mathrm{~Pa}^{-1}\right]\end{array}$}} & \multicolumn{2}{|c|}{$M W D=1.11$} & \multirow{2}{*}{\multicolumn{2}{|c|}{$\begin{array}{c}M W D=1.04 \\
J_{\rho}^{0}=1.16 \times 10^{-5}\left[\mathrm{~Pa}^{-1}\right]\end{array}$}} & \multirow{2}{*}{\multicolumn{2}{|c|}{$M W D=1.06$}} \\
\hline & & \multirow{2}{*}{\multicolumn{2}{|c|}{$\begin{array}{c}J_{e}^{0}=1.15 \times 10^{-5}\left[\mathrm{~Pa}^{-1}\right] \\
\eta_{0}=8.13 \times 10^{6}[\mathrm{~Pa} \mathrm{~s}]\end{array}$}} & & & & \\
\hline \multicolumn{2}{|c|}{$\eta_{0}=8.65 \times 10^{5}[\mathrm{~Pa} \mathrm{~s}]$} & & & \multicolumn{2}{|c|}{$\eta_{0}=8.26 \times 10^{7}[\mathrm{~Pa} \mathrm{~s}]$} & \multicolumn{2}{|c|}{$\eta_{0}=7.57 \times 10^{8}[\mathrm{~Pa} \mathrm{~s}]$} \\
\hline \multicolumn{2}{|c|}{$\tau_{R}=6.4 \mathrm{~s}$} & \multicolumn{2}{|c|}{$\tau_{R}=22.9 \mathrm{~s}$} & \multicolumn{2}{|c|}{$\tau_{R}=91.5 \mathrm{~s}$} & \multicolumn{2}{|c|}{$\tau_{R}=329 \mathrm{~s}$} \\
\hline \multicolumn{2}{|c|}{$\tau_{w}=5.0 \mathrm{~s}$} & \multicolumn{2}{|c|}{$\tau_{w}=94 \mathrm{~s}$} & \multicolumn{2}{|c|}{$\tau_{w}=961 \mathrm{~s}$} & \multicolumn{2}{|c|}{$\tau_{w}=11351 \mathrm{~s}$} \\
\hline \multicolumn{2}{|c|}{$\tau_{a}=141 \mathrm{~s}$} & \multicolumn{2}{|c|}{$\tau_{a}=203 \mathrm{~s}$} & \multicolumn{2}{|c|}{$\tau_{a}=384 \mathrm{~s}$} & \multicolumn{2}{|c|}{$\tau_{a}=1462 \mathrm{~s}$} \\
\hline$g_{i}[\mathrm{~Pa}]$ & $\lambda_{i}[\mathrm{~s}]$ & $g_{i}[\mathrm{~Pa}]$ & $\lambda_{i}[\mathrm{~s}]$ & $g_{i}[\mathrm{~Pa}]$ & $\lambda_{i}[\mathrm{~s}]$ & $g_{i}[\mathrm{~Pa}]$ & $\lambda_{i}[\mathrm{~s}]$ \\
\hline $9.15 \times 10^{6}$ & $2.91 \times 10^{-3}$ & $4.10 \times 10^{5}$ & $6.73 \times 10^{-2}$ & $2.38 \times 10^{5}$ & $4.75 \times 10^{-1}$ & $4.63 \times 10^{5}$ & $1.04 \times 10^{-1}$ \\
\hline $3.71 \times 10^{5}$ & $9.61 \times 10^{-2}$ & $1.02 \times 10^{7}$ & $3.06 \times 10^{-3}$ & $3.71 \times 10^{4}$ & $5.29 \times 10^{0}$ & $7.79 \times 10^{4}$ & $1.07 \times 10^{0}$ \\
\hline $1.40 \times 10^{5}$ & $9.85 \times 10^{-1}$ & $1.47 \times 10^{5}$ & $4.77 \times 10^{-1}$ & $2.71 \times 10^{4}$ & $1.96 \times 10^{1}$ & $3.00 \times 10^{4}$ & $9.70 \times 10^{0}$ \\
\hline \multirow[t]{5}{*}{$1.05 \times 10^{5}$} & $6.33 \times 10^{0}$ & $7.73 \times 10^{4}$ & $3.40 \times 10^{0}$ & $5.46 \times 10^{4}$ & $9.20 \times 10^{1}$ & $2.55 \times 10^{4}$ & $5.16 \times 10^{1}$ \\
\hline & & $6.77 \times 10^{4}$ & $1.93 \times 10^{1}$ & $4.96 \times 10^{4}$ & $4.43 \times 10^{2}$ & $3.33 \times 10^{4}$ & $2.35 \times 10^{2}$ \\
\hline & & $7.39 \times 10^{4}$ & $8.51 \times 10^{1}$ & $4.33 \times 10^{4}$ & $1.26 \times 10^{3}$ & $4.31 \times 10^{4}$ & $1.14 \times 10^{3}$ \\
\hline & & $1.11 \times 10^{2}$ & $1.35 \times 10^{3}$ & & & $5.15 \times 10^{4}$ & $5.97 \times 10^{3}$ \\
\hline & & & & & & $2.28 \times 10^{4}$ & $1.71 \times 10^{4}$ \\
\hline
\end{tabular}

tions, or equivalently that the tension in the deformed macromolecular chain remains constant and equal to its equilibrium value [Doi and Edwards (1986)]. The main contribution to the extra stress tensor $\sigma(t)$ is then given by the orientation of the tube segments due to the flow. The resulting constitutive equation is of the single integral form,

$$
\sigma(t)=\int_{-\infty}^{t} m\left(t-t^{\prime}\right) \mathbf{S}_{\mathrm{DE}}^{\mathrm{IA}}\left(t, t^{\prime}\right) d t^{\prime}
$$

if the tube segments are assumed to align independently of each other in the flow field [the "independent alignment (IA)" approximation]. $m\left(t-t^{\prime}\right)$ is the memory function with $m\left(t-t^{\prime}\right)=\sum_{i} \frac{g_{i}}{\lambda_{i}} e^{-\left(t-t^{\prime}\right) / \lambda_{i}}$, and the relative strain measure $\mathbf{S}_{\mathrm{DE}}^{\mathrm{IA}}$ is given by

$$
\mathbf{S}_{\mathrm{DE}}^{\mathrm{IA}}\left(t, t^{\prime}\right) \equiv 5\left\langle\frac{\mathbf{u}^{\prime} \mathbf{u}^{\prime}}{u^{\prime 2}}\right\rangle_{0}=5 \mathbf{S}\left(t, t^{\prime}\right),
$$

where $\mathbf{S}$ is the relative second-order orientation tensor. The bracket denotes an average over an isotropic distribution of unit vectors $\mathbf{u}\left(t^{\prime}\right)$ at time $t^{\prime}$, and can be expressed as a surface integral over the unit sphere,

$$
\langle\rangle_{0} \equiv \frac{1}{4 \pi} \oiint[] \sin \theta_{o} d \theta_{o} d \varphi_{o} .
$$

At time $t$, the unit vectors are deformed to vectors $\mathbf{u}^{\prime}$, which are calculated from the affine deformation hypothesis (with $\mathbf{F}^{-1}\left(t, t^{\prime}\right)$ as the relative deformation gradient tensor) as

$$
\mathbf{u}^{\prime}\left(t, t^{\prime}\right)=\mathbf{F}^{-1}\left(t, t^{\prime}\right) \cdot \mathbf{u}\left(t^{\prime}\right),
$$

where $u^{\prime}$ indicates the length of the vector $\mathbf{u}^{\prime}$. 


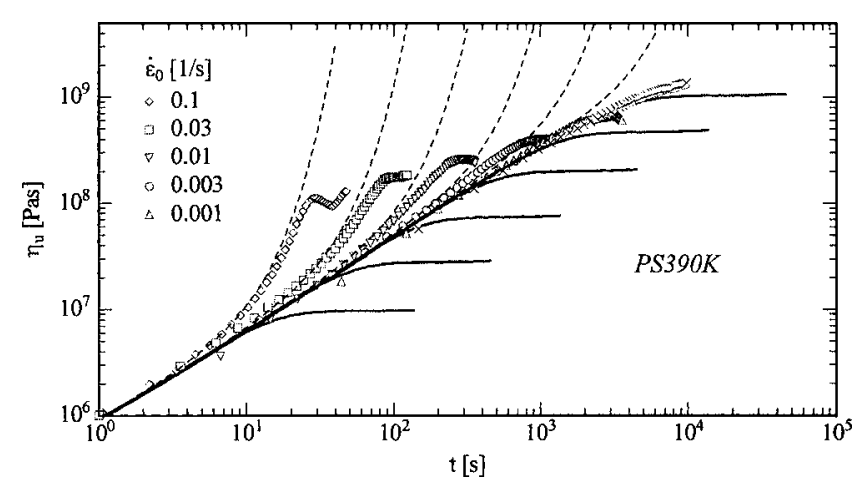

FIG. 1. Comparison of measured transient elongational viscosity data of PS390K (symbols) to predictions by DE theory (full lines) and assumption of affine chain stretch (dotted lines).

As shown by Currie [Currie (1982)], the strain energy function of the DE model is given by

$$
\frac{w_{\mathrm{DE}}}{3 k T}=\left\langle\ln u^{\prime}\right\rangle_{0}
$$

which represents the free rotational energy of a tube segment. Consequently, the DE model does not account for any strain hardening. This is demonstrated in Figs. 1 and 2, where predictions of the DE model are compared to start-up and steady-state elongational data of PS390K. As expected, predictions and data deviate increasingly with increasing strain rate. However, Fig. 1 reveals the interesting fact that the experimentally determined steady-state elongational viscosity is reached at the same time, when the orientation saturates as described by the DE model.

Doi and Edwards [Doi and Edwards (1986)] incorporated a stretch process with a stretch $\lambda$ of the tube segments due to the flow in order to explain the discrepancies of the DE theory at the startup of shear and extensional flows. Preaveraging the stretch, i.e., assuming that the stretch is uniform along the chain contour length and an explicit function $\lambda(t)$ of the observation time, which operates on the orientational configuration resulting from the integration over the strain history, the extra stress tensor is given by

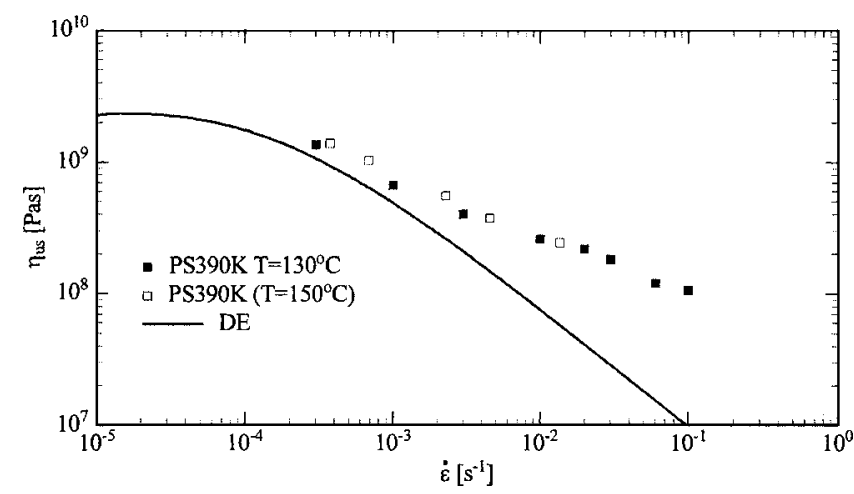

FIG. 2. Comparison of steady-state elongational viscosity data of PS390K measured at $130{ }^{\circ} \mathrm{C}$ (full symbols), and at $150{ }^{\circ} \mathrm{C}$ (shifted to $130^{\circ} \mathrm{C}$, open symbols), to predictions by DE theory (full line). 


$$
\boldsymbol{\sigma}(t)=\lambda^{2}(t) \int_{-\infty}^{t} m\left(t-t^{\prime}\right) \mathbf{S}_{\mathrm{DE}}^{\mathrm{IA}}\left(t, t^{\prime}\right) d t^{\prime}
$$

Equation (8) generated the necessity to find a stretch evolution equation, and a vast variety of concepts based on different kinetic ideas have been proposed in recent years [see, e.g., Doi (1980); Pearson et al. (1989); McLeish et al. (1998); Mead et al. (1998)].

\section{AFFINE CHAIN STRETCH AND THE ROUSE TIME OF THE CHAIN}

While in models with preaveraged stretch, the tube diameter is invariably assumed to stay constant and equal to its equilibrium value $a_{0}$, stretch can also be introduced by the assumption of a strain-dependent tube diameter, as first suggested by Marrucci and de Cindio [Marrucci and de Cindio (1980)]. In this way, also the preaveraging of the stretch can be avoided, which is inherently present in models based on Eq. (8) or its differential approximations.

A generalized tube model with strain-dependent tube diameter was presented by Wagner and Schaeffer [Wagner and Schaeffer (1992, 1993, 1994)]. In the molecular stress function (MSF) theory, tube stretch is directly related to the tube diameter $a$, which decreases from its equilibrium value $a_{0}$ with increasing stretch. Taking into account that the tube diameter $a$ represents the mean field of the surrounding chains, it is assumed that the tube diameter is independent of the orientation of tube segments. The extra stress is then given as

$$
\boldsymbol{\sigma}(t)=\int_{-\infty}^{t} \mathrm{~m}\left(t-t^{\prime}\right) f^{2} \mathbf{S}_{\mathrm{DE}}^{\mathrm{IA}}\left(t, t^{\prime}\right) d t^{\prime},
$$

where the MSF $f=f\left(t, t^{\prime}\right)$ is the inverse of the relative tube diameter,

$$
f\left(t, t^{\prime}\right)=a_{0} / a\left(t, t^{\prime}\right) .
$$

In contrast to Eq. (8), stretch in Eq. (9) does not only depend on the observation time $t$, but also on the strain history, i.e., for time-dependent strain histories, chain segments with long relaxation times (i.e., at the center of the chain) see higher stretches than chain segments with short relaxation times (i.e., at the chain ends). by

Assuming now (on average) affine stretch, the evolution equation for $f$ is simply given

$$
\frac{\partial f}{\partial t}=f(\kappa: \mathbf{S}),
$$

with velocity gradient $\kappa$, which can be integrated to

$$
f=e^{\left\langle\ln u^{\prime}\right\rangle_{0}}
$$

Realizing that the power input of the stress tensor per chain segment corresponds to the change in the free energy $w_{\mathrm{MSF}}$ of a chain segment,

$$
\frac{1}{3 k T} \frac{\partial w_{\mathrm{MSF}}}{\partial t}=f^{2}(\kappa: \mathbf{S})
$$

this corresponds to a strain energy function of the form 


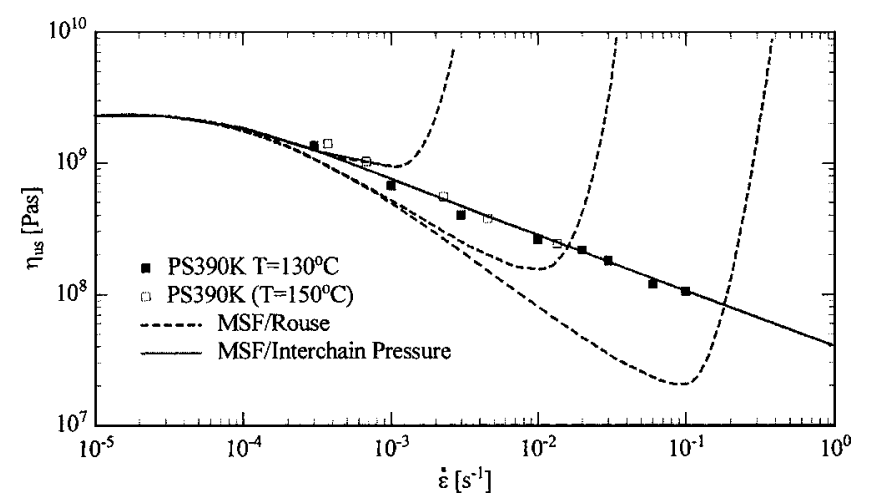

FIG. 3. Comparison of measured steady-state elongational viscosity data of PS390K (symbols) to predictions by MSF model with chain stretch balanced by Rouse-type spring force (dotted lines, from left to right: $\tau_{R}$ $=329 \mathrm{~s} / 32.9 \mathrm{~s} / 3.29 \mathrm{~s}$ ) and by interchain pressure (full line, $\tau_{a}=1462 \mathrm{~s}$ ).

$$
\frac{w_{\mathrm{MSF}}}{3 k T}=\frac{1}{2}\left(f^{2}-1\right)
$$

i.e., the chain segments behave as linear springs. As shown in Fig. 1, the assumption of affine chain stretch, although unbound, describes the onset of strain hardening quantitatively, at least at higher strain rates.

Following conventional arguments [e.g. Pearson et al. (1989)], we now assume that the affine chain deformation is balanced by the linear spring force, i.e., the evolution equation for $f$ takes the form

$$
\frac{\partial f}{\partial t}=f(\kappa: \mathbf{S})-\frac{1}{\tau_{R}}(f-1),
$$

where $\tau_{R}$ is the Rouse time of the chain. For PS390K, a value of $\tau_{R}=329 \mathrm{~s}$ is obtained from Eq. (1). As demonstrated in Fig. 3, this clearly does not describe the steady-state elongational viscosity of PS390K. As the exact value of the Rouse time may depend on the method applied, we also show the predictions for $\tau_{R} / 10$ and $\tau_{R} / 100$. It is obvious from Fig. 3 that a linear spring force will quench chain stretch as long as the product of $\dot{\varepsilon} \tau_{R}$ is much smaller than 1 , while chain stretch will diverge in the limit of $\dot{\varepsilon} \tau_{R} \rightarrow 1$, resulting in a diverging steady-state elongational viscosity. This is clearly seen by setting the left-hand side of Eq. (15) to zero, which gives the maximum stretch $f_{\max }$ as

$$
f_{\max }=\frac{1}{1-\dot{\varepsilon} \tau_{R}} .
$$

(We will not discuss finite extensibility here, which is usually invoked to rectify the situation to some extent and to remove the singularity.)

\section{THE INTERCHAIN PRESSURE TERM}

Considering a chain composed of $N$ Kuhn segments of length $b$, confined within a box of dimensions $L_{x}, L_{y}$, and $L_{z}$, where the overall length of the chain is much larger than the dimensions of the confining box, one can write the pressure acting on the box wall normal to the $x$ axis as the gradient of free energy $A$ in the $x$ direction [Doi and Edwards 1986], 


$$
p_{x}=-\frac{1}{L_{y} L_{z}} \frac{\partial A}{\partial L_{x}}=\frac{\pi^{2} N b^{2}}{3 L_{x}^{2}} \frac{k_{B} T}{V} .
$$

With $L_{x} \approx L_{y} \approx a$ and $V=a^{2} L_{\text {Tube }}$ this leads to [Marrucci and Ianniruberto (2004)]

$$
p_{x}=p_{y} \approx k T \frac{N b^{2}}{a^{4} L_{\text {Tube }}} .
$$

As $N b^{2}$ is a constant, and considering that the product $a L_{\text {Tube }}$ is constant even at large deformations [see, e.g., Wagner and Schaeffer (1992)], the relative radial pressure $p / p_{0}$ will increase inversely proportional to the third power of the tube diameter $a$ from its equilibrium value $p_{0}$,

$$
\frac{p}{p_{0}}=\frac{a_{0}^{3}}{a^{3}} .
$$

Marrucci and Ianniruberto assumed that this radial pressure increase is balancing the tube diameter reduction, and they derived the following evolution equation for the tube diameter $a$ [Marrucci and Ianniruberto (2004)],

$$
\frac{\partial a}{\partial t}=-\dot{\varepsilon} a+\frac{a_{0}}{\tau_{a}}\left(\frac{a_{0}^{3}}{a^{3}}-1\right) .
$$

We call $\tau_{a}$ the tube diameter relaxation time.

We now replace the first term on the right-hand side of Eq. (20) by the general tensorial description in analogy to Eq. (11) above [see, e.g., Wagner et al. (2001)], which leads to:

$$
\frac{\partial a}{\partial t}=-(\kappa: \mathbf{S}) a+\frac{a_{0}}{\tau_{a}}\left(\frac{a_{0}^{3}}{a^{3}}-1\right)
$$

Inserting the definition of the MSF, $f=\frac{a_{0}}{a}$, and considering that $\frac{\partial a}{\partial t}$ can be expressed as $\frac{\partial a}{\partial t}=-a_{0} \frac{1}{f^{2}} \frac{\partial f}{\partial t}$, we obtain from Eq. (21) the following evolution equation for the tension in a chain segment:

$$
\frac{\partial f}{\partial t}=f\left[(\kappa: \mathbf{S})-\frac{f\left(f^{3}-1\right)}{\tau_{a}}\right] .
$$

Equation (22) together with Eq. (9) represents a full constitutive equation with only one parameter, the tube diameter relaxation time $\tau_{a}$.

The maximum stretch $f_{\max }$ is reached, when the orientation saturates, i.e., when $\boldsymbol{\kappa}: \mathbf{S}$ $\rightarrow \dot{\varepsilon}$. Due to the nonlinear restoring pressure term on the right-hand side of Eq. (22), the divergence of the maximum stretch $f_{\max }$ in constant strain-rate elongation is now removed, and $f_{\max }$ is given by

$$
f_{\max }\left(f_{\max }^{3}-1\right)=\dot{\varepsilon} \tau_{a}
$$

or for $f_{\text {max }} \gg 1$

$$
f_{\max }^{2} \approx \sqrt{\dot{\varepsilon} \tau_{a}}
$$

For fast deformations, the extensional stress $\sigma$ is proportional to $f_{\max }^{2}$,

$$
\sigma \propto f_{\max }^{2}
$$

and, therefore, the steady-state elongational viscosity scales with 


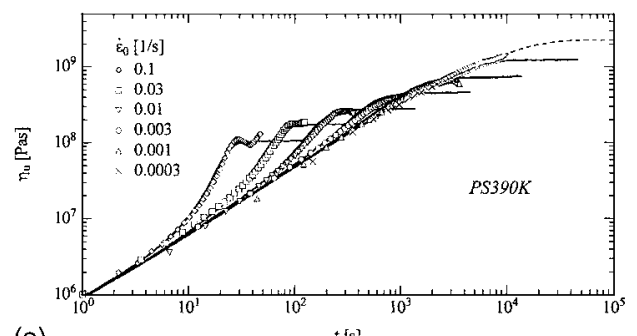

(a)

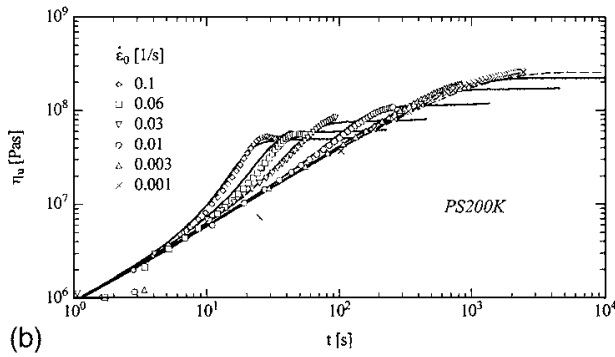

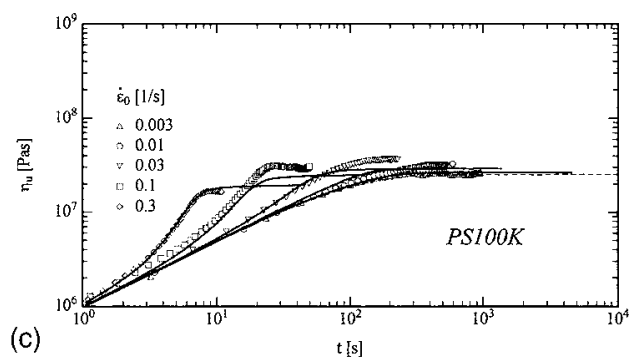

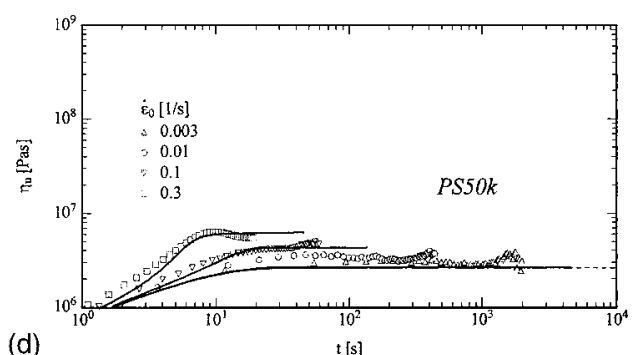

FIG. 4. Comparison of measured transient elongational viscosity data to predictions by MSF model with chain stretch balanced by interchain pressure: (a) PS390K, $\tau_{a}=1462 \mathrm{~s}$; (b) PS200K, $\tau_{a}=384 \mathrm{~s}$; (c) PS100K, $\tau_{a}$ $=203 \mathrm{~s}$; and (d) PS50K, $\tau_{a}=141 \mathrm{~s}$.

$$
\eta_{\mathrm{us}}=\frac{\sigma}{\dot{\varepsilon}} \propto \frac{\sqrt{\dot{\varepsilon} \tau_{a}}}{\dot{\varepsilon}}=\sqrt{\tau_{a}} \dot{\varepsilon}^{-1 / 2}
$$

which corresponds to the results of Marrucci and Ianniruberto [Marrucci and Ianniruberto (2004)]. However, due to the width of the relaxation spectra even for nearly monodisperse melts (see Table I), the actual decrease of the viscosity observed is smaller than predicted by Eq. (26), and is closer to a power of -0.4 . As shown in Fig. 3, the steadystate elongational viscosity as predicted from Eqs. (9) and (22) for PS390K with the value of the tube diameter relaxation time fitted to $\tau_{a}=1462 \mathrm{~s}$ is in excellent agreement with the data.

Figures 4(a)-4(d) present comparisons of the transient elongational viscosity data of Bach et al. [Bach et al. (2003)] and Hassager [Hassager (2004)] to predictions of Eqs. (9) and (22). The time-dependent increase, including the plateau value of the elongational viscosities, is well modeled for all four PS samples by fitting the tube diameter relaxation time $\tau_{a}$ to the values given in Table I. Slight deviations between data and predictions are only seen for some elongation rates in the case of PS100K.

In Fig. 5, the steady-state elongational viscosities of all samples as calculated from Eqs. (9) and (22) are presented and compared to the experimental data, and excellent agreement is observed. For PS100K and PS50K, a maximum in the steady-state elongational viscosity is found. For these two melts, the tube diameter relaxation time $\tau_{a}$ is larger than the reptation time $\tau_{w}$ as shown in Fig. 6, where tube relaxation time $\tau_{a}$, the reptation time $\tau_{w}$, and the Rouse time $\tau_{R}$ are plotted as a function of molar mass. This means that chain stretch is already significant at elongation rates which are smaller than the inverse reptation time. Figure 6 also reveals that for the two PSs with the highest molar masses, PS390K and PS200K, the tube diameter relaxation time $\tau_{a}$ seems to scale in a similar way as the Rouse time $\tau_{R}$, i.e., 


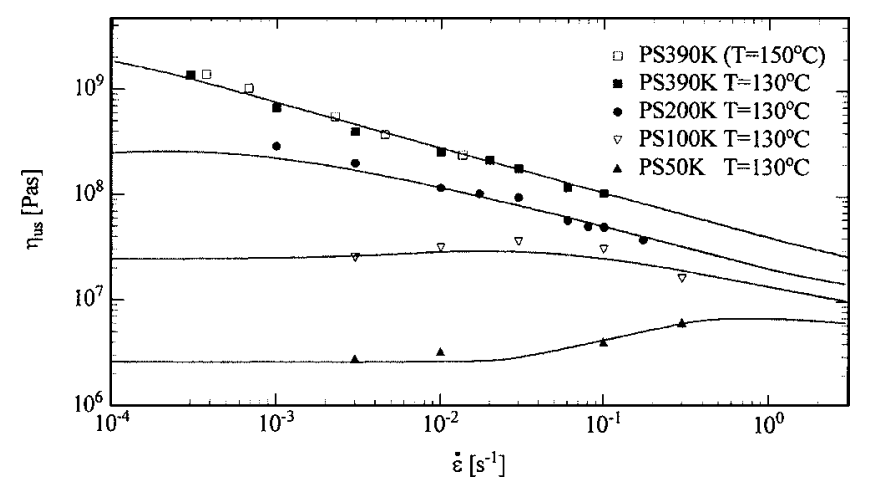

FIG. 5. Comparison of measured steady-state elongational viscosity data of four narrow molar mass distribution PS melts (symbols) to predictions of MSF model with chain stretch balanced by interchain pressure (lines).

$$
\tau_{a} \propto M^{2}
$$

and $\tau_{a}$ is about 4.4 times larger than $\tau_{R}$, while for the two lower molar masses, PS100K and PS50K, a scaling of the order

$$
\tau_{a} \propto M^{1 / 2}
$$

seems to be appropriate. An argument in favor of the latter relation might be that for melts with few (some three to eight) entanglements, where the difference between reptation time and Rouse time becomes small or even vanishes as for PS50K, the radius of

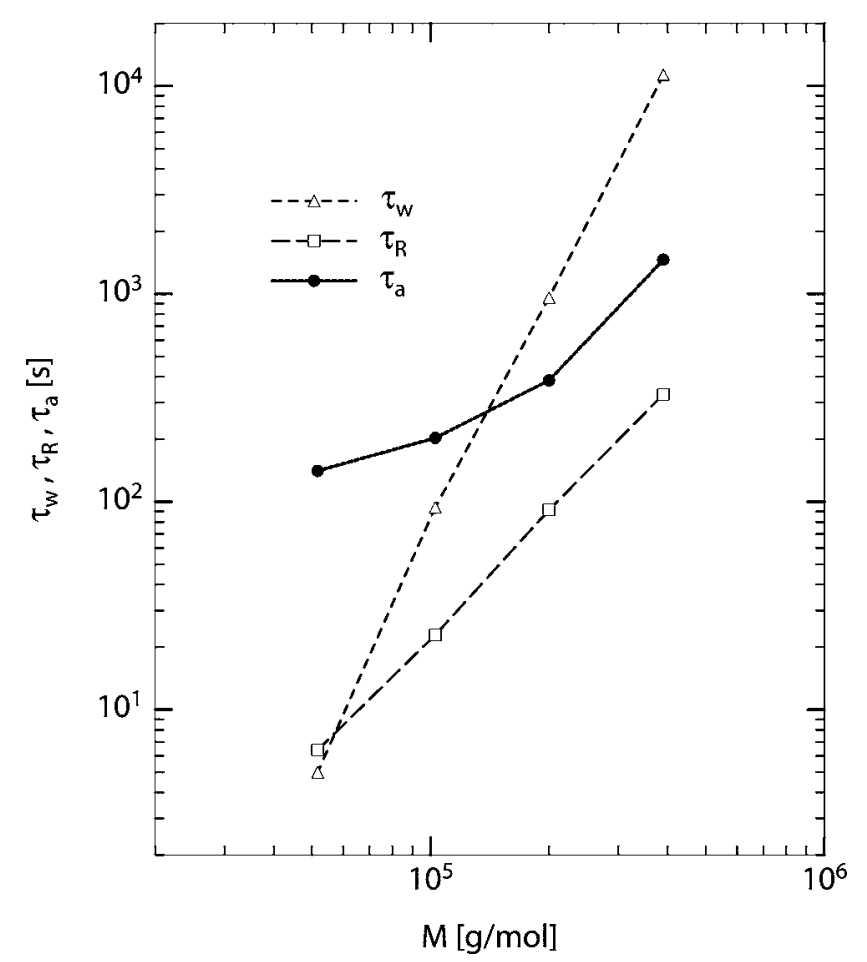

FIG. 6. Reptation time $\tau_{w}$, Rouse time $\tau_{R}$, and tube diameter relaxation time $\tau_{a}$ as a function of molar mass M. 
gyration of the coil might be more important for the tube diameter relaxation than the length of the chain. One may argue that the tube concept becomes vague for melts with only a few entanglements, but it should be noted that the reptation time $\tau_{w}$ still shows the expected scaling, i.e., $\tau_{w} \sim M^{3.6}$.

\section{CONCLUSIONS}

The elongational behavior of narrow molar mass distribution PS melts as determined previously [Bach et al. (2003) and Hassager (2004)] can be explained quantitatively by assuming affine chain stretch, which is balanced by interchain pressure as introduced by Marrucci and Ianniruberto [Marrucci and Ianniruberto (2004)]. These ideas can readily be introduced into the MSF concept allowing for a variation of the tube diameter, resulting in a single integral constitutive equation for the stress tensor, and an evolution equation for the tension in the chain. The interchain pressure, which is inverse proportional to the third power of the tube diameter $a$, represents a force that is restoring the tube diameter to is equilibrium value $a_{0}$, and is governed by a tube diameter relaxation time $\tau_{a}$. The nonlinearity of the pressure term is essential for removing the divergence of the chain stretch caused by the Rouse time catastrophe, and for explaining the scaling of the steady-state elongational viscosity observed. As the tube diameter relaxation time $\tau_{a}$ represents the relaxation of topological constraints caused by many surrounding chains and is therefore the result of a highly cooperative effect, it is perhaps not surprising that it is found to be larger than the Rouse time of the chain. For PS melts with more than ten entanglements per chain, $\tau_{a}$ seems to scale with the square of the molar mass, while for melts with fewer entanglements, a dependence closer to a power of 0.5 is found. Further experimental investigations are needed to confirm these scaling relations.

\section{References}

Bach, A., K. Almdal, H. K. Rasmussen, and O. Hassager, "Elongational viscosity of narrow molar mass distribution polystyrene," Macromolecules 36, 5174-5179 (2003).

Bhattacharjee, P. K., J. P. Oberhauser, G. H. McKinley, L. G. Leal, and T. Sridhar, "Extensional rheometry of entangled solutions," Macromolecules 35, 10131-10148 (2002).

Bhattacharjee, P. K., D. A. Nguyen, G. H. McKinley, and T. Sridhar, "Extensional stress growth and stress relaxation in entangled polymer solutions," J. Rheol. 47, 269-290 (2003).

Currie, P. K., "Constitutive equations for polymer melts predicted by the Doi-Edwards and Curtiss-Bird kinetic theory models," J. Non-Newtonian Fluid Mech. 11, 53-68 (1982).

Doi, M., "A constitutive equation derived from the model of Doi and Edwards for concentrated polymer solutions and polymer melts, J. Polym. Sci., Polym. Phys. Ed. 18, 2055-2067 (1980)

Doi, M., and S. F. Edwards, The Theory of Polymer Dynamics (Oxford University Press, Oxford, 1986).

Fang, J., M. Kröger, and H. C. Öttinger, "A thermodynamically admissible reptation model for fast flows of entangled polymers. II. Model predictions for shear and extensional flows," J. Rheol. 44, 1293-1317 (2000).

Hassager, O., "Polymer fluid mechanics: Molecular orientation and stretching," Proc. XIVth International Congress on Rheology, NF01 (2004).

Isaki, T., M. Takahashi, and O. Urakawa, "Biaxial damping function of entangled monodisperse polystyrene melts: Comparison with the Mead-Larson-Doi model," J. Rheol. 47, 1201-1210 (2003).

Marrucci, G., and B. de Cindio, "The stress relaxation of molten PMMA at large deformations and its theoretical interpretation,” Rheol. Acta 19, 68-75 (1980).

Marrucci, G., and G. Ianniruberto, "Interchain pressure effect in extensional flows of entangled polymer melts," Macromolecules 37, 3934-3942 (2004). 
McKinley, G., and T. Sridhar, "Filament-stretching rheometry of complex fluids," Annu. Rev. Fluid Mech. 34, 375-415 (2002).

McLeish, T. C. B., and R. G. Larson, "Molecular constitutive equations for a class of branched polymers: the pom-pom polymer," J. Rheol. 42, 81-110 (1998).

Mead, D. W., D. Yavich, and L. G. Leal, "The reptation model with segmental stretch II. Steady-state properties," Rheol. Acta 34, 360-383 (1995).

Mead, D. W., R. G. Larson, and M. Doi, "A molecular theory for fast flows of entangled polymers," Macromolecules 31, 7895-7914 (1998).

Osaki, K., K. Nishizawa, and M. Kurata, "Material time constant characterizing the nonlinear viscoelasticity of entangled polymeric systems," Macromolecules 15, 1068-1071 (1982).

Pearson, D. S., A. Kiss, L. Fetters, and M. Doi, "Flow-induced birefringence of concentrated polyisoprene solutions," J. Rheol. 33, 517-535 (1989).

Schweizer, T., "The uniaxial elongational rheometer RME-Six years of experience,” Rheol. Acta 39, 428-443 (2000).

Takahashi, M., T. Isaki, T. Takigawa, and T. Masuda, "Measurement of biaxial and uniaxial extensional flow behavior of polymer melts at constant strain rates," J. Rheol. 37, 827-846 (1993).

Wagner, M. H., and J. Schaeffer, "Nonlinear strain measures for general biaxial extension of polymer melts," J. Rheol. 36, 1-26 (1992).

Wagner, M. H., and J. Schaeffer, "Rubbers and polymer melts: Universal aspects of nonlinear stress-strain relations," J. Rheol. 37, 643-661 (1993).

Wagner, M. H., and J. Schaeffer, "Assessment of nonlinear strain measures for extensional and shearing flows of polymer melts,” Rheol. Acta 33, 506-516 (1994).

Wagner, M. H., P. Rubio, and H. Bastian, "The molecular stress function model for polydisperse and polymer melts with dissipative convective constraint release," J. Rheol. 45, 1387-1412 (2001).

Winter, H. H., and M. Mours, IRIS Development, Amherst, MA (2003). 